\title{
Effect of Antenatal Instructional Package on Pregnant Women' Knowledge, Attitude and Practices
}

\author{
Amira Mohammed Salama*, Fatma Kamal Aly \\ Obstetrics and Woman's Health Nursing, Faculty of Nursing, Benha University, Egypt \\ *Corresponding author: amirasalama88@yahoo.com \\ Received April 05, 2019; Revised May 14, 2019; Accepted May 24, 2019
}

\begin{abstract}
Background: Antenatal care is a careful, systematic assessment and follow-up of pregnant women that includes education, counseling, screening, and treatment to assure the best possible health of the mother and fetus. Aim of the research was to examine the effect of antenatal instructional package on pregnant women' knowledge, attitude and practices. Design: A quasi-experimental research design was adopted to fulfil the aim of this research. Setting: The research was conducted at obstetrics \& gynaecological outpatient clinic in Benha university hospital. Sample: A purposive sample of one hundred and forty pregnant women among those attending the above mentioned setting. Tools: Data were collected through five main tools: A Structured interviewing questionnaire, maternal knowledge questionnaire, maternal health attitude questionnaire, maternal health practices questionnaire, and women's satisfaction sheet. Results: showed that nearly one third of studied women in both study group (41.4\%) and control group (37.1\%) had source of information from friends and families while minority of them had source of information from newspaper. Also there was no statistical significant difference regarding all knowledge, attitude and practice items about antenatal care between the study and the control group before instructional package implementation. However, a highly statistically significant difference (p-values $<0.001$ ) was observed between the study and the control group one month after instructional package implementation. Additionally total knowledge, total attitude and total practices regarding antenatal care were greatly improved after instructional package implementation than pre implementation among study group while there were minimal improvements after instructional package implementation than pre implementation among control group. Also the majority of pregnant women in the study group were satisfied with the antenatal instructional package implementation. Conclusion: the study concluded that research hypotheses are supported and the antenatal instructional package had appositive effect on improvement of pregnant women' knowledge and in turn attitude and practices were also improved in study group compared to control group .Also the majority of pregnant women in the study group were satisfied with the antenatal instructional package implementation. Recommendations: From time to time, the antenatal instructional package should be restructured and reviewed to meet up with the health needs of the pregnant women.
\end{abstract}

Keywords: antenatal, instructional package, pregnant women,

Cite This Article: Amira Mohammed Salama, and Fatma Kamal Aly, "Effect of Antenatal Instructional Package on Pregnant Women' Knowledge, Attitude and Practices.” American Journal of Nursing Research, vol. 7, no. 4 (2019): 519-533. doi: 10.12691/ajnr-7-4-14.

\section{Introduction}

Maternal morbidity and mortality constitute a serious health problem that requires systematic investigations. Antenatal care (ANC) is an important determinant of good maternal health. Lack of ANC is a risk factor for maternal mortality, and reduction of maternal mortality requires early detection of high risk pregnancies through proper ANC [1]. Confidential inquiries into maternal deaths in developing countries have found a positive association with inadequate antenatal care as a risk factor for maternal mortality [2].

Antenatal care is considered as a back bone of obstetrical services, it is substantial for health of pregnant women and it is the way in which maternal and fetal complications are determined and managed. Good caring during pregnancy is so important for the health of the mother and the development of the unborn baby, it links the woman and family with a formal health system which will increase the chance of using a skilled attendant particularly at birth and contributes to well health through the life cycle [2].

Antenatal care is a type of preventative care with the goal of providing regular check-ups that allow health care providers identify, treat and prevent potential health problems throughout the course of the pregnancy while promoting healthy lifestyles that benefit both mother and child [3]. The availability of routine antenatal care has played a part in reducing maternal death rates and miscarriages as well as birth defects, low birth weight, and other preventable health problems. At the initial antenatal care visit and with the aid of a special booking checklist 
the pregnant women become classified as either normal risk or high risk [4].

Antenatal care consists of a routine number of scheduled visits aiming at screening and detection of conditions likely to increase adverse effects during pregnancy to ensure healthy outcomes for women and newborn; provide therapeutic interventions and timely treatment of possible complications in pregnancy; and educate pregnant women about planning for safe birth, emergencies during pregnancy, and how to deal with these. [5]. Through the focused antenatal care approach, WHO recommends four ANC visits in low-risk pregnancies, with the first visit in the first trimester, ideally before 12 weeks, but no later than 16 weeks, and the second, third, and fourth visits at 24-28, 32, and 36 weeks, respectively. [6].

Roughly, a thousand mothers all around the world die daily due to pregnancy and delivery related causes. [7], which resemble $25 \%$ of maternal deaths according to World Health Organization. Although, maternal mortality rates in the past 25 years has decreased by about $44 \%$, it is still considered by WHO to be intolerably immense. The vast majority of these fatalities take place in low-resource settings, and hence preventable in a great measure. This matter of concern, therefore, was a first priority in the Millennium Development Goals acknowledged by the international community in 2000 which obligate the medical community globally to promote maternal health as one of their recommendations [8].

Early entry to ANC is important for early detection and treatment of adverse pregnancy-related outcomes as it provides expecting mothers with information that enable them to identify potential warning signs of malfunction or abnormalities during pregnancy as well as strategies to adhere to prescribed treatments and referrals. It has been shown that approximately $80 \%$ of maternal mortality could be prevented if the affected mother had a timely access to the essential maternity and basic health-care services [9].

Health knowledge is an important element to enable women to be aware of health status and the importance of appropriate ANC as satisfaction of the women seeking help, is one of the most important qualitative indexes of health care provision, and has a very special importance in prenatal care. Satisfying pregnant women is achieved through satisfying needs and expectations [10].

The need of implementing knowledge, attitude and practice of ANC intervention in pregnant women it has been showed that as a package comprising the following interlocking system includes interventions, early screening, administration of a preventive prophylactic therapy and curative of the various detected risk conditions effectively on the basis of reduced maternal complications [11].

Health care provider consider ANC as an important opportunity for discussing a pregnant women about health behaviour during pregnancy and about recognizing complications that may arise during pregnancy through screening for conditions likely to increase adverse outcomes, providing therapeutic interventions known to be beneficial, and educating pregnant women about planning for a safe birth, emergencies during pregnancy, and how to deal with them [12].

Nurses during antenatal care could provide pregnant women with valuable information and advices that could help pregnant women during pregnancy and delivery to avoid high rates of death that result from inappropriate knowledge about antenatal care [13]. During antenatal period, supplementing pregnant women with folic acid, Calcium and important vitamins as well as providing the mother with care and information could assist the family to overcome pregnancy complications and promotion of breast feeding [14].

\subsection{Significance of the Research}

Each year, approximately one third of a million women worldwide die due to pregnancy related conditions; 99\% of these deaths occur in developing countries of Africa and Asia and approximately three quarter of them are considered avoidable [15]. Preventing problems for mothers and babies depends on an operational continuum of care with accessible high quality care before and during pregnancy, childbirth and the postnatal period, and this also depends on the support available to help pregnant women reach services particularly when complications occur [16].

\subsection{Aim of the Research}

This research aims to examine the effect of antenatal instructional package on pregnant women' knowledge, attitude and practices. This aim will be achieved through the following objectives:

1. Assessing knowledge, attitude and practices of pregnant women regarding antenatal care.

2. Designing and implementing antenatal instructional package.

3. Evaluating the outcome of antenatal instructional package.

\subsection{Research Hypotheses}

H1- Pregnant women who will receive antenatal instructional package will exhibit better knowledge, attitude and practices than those who won't.

H2- Pregnant women of study group will be satisfied with antenatal instructional package.

\section{Subjects and Method}

\subsection{Research Design}

A quasi-experimental research design was used (time series design) pre/post-test, two groups were studied.

\subsection{Setting}

This research was conducted at obstetrics \& gynaecological outpatient clinic in Benha university hospital which includes one room divided into diagnostic and examination areas. As well as, waiting area for women admission where the researchers interviewed the recruited women to implement the antenatal instructional package. This clinic provides services of obstetrics and gynaecological care, family planning counseling and any outpatient procedures. It started from 9am to $12 \mathrm{pm}$. 


\subsection{Sampling}

A purposive sample of (140) pregnant women among those attending the above mentioned setting for a period of six months. Women were randomly divided into two groups (control group $=70$ women who received routine care and study group $=70$ women who received the antenatal instructional package). The studied sample was selected according to the following inclusion criteria: The woman must be confirmed pregnant and agree to participate in the study, women attending antenatal clinic at first trimester of pregnancy, women can read and write and women with no pregnancy complications and free from any medical disorders.

\subsection{Tools of Data Collection}

Data were collected through five main tools.

\subsubsection{First Tool}

- A Structured interviewing questionnaire.

It was designed by the researchers after reviewing related literature $[14,17,18]$, it was written in an Arabic language. It was consisted of two parts:

The first part: - Socio-demographic data of women, it consisted of (age, residence, level of education, occupation, income and transportation to access health facility).

The second part: - Obstetrical history of women, it consisted of (gestational age, No. of pregnancies).

\subsubsection{Second Tool}

\section{- Maternal Knowledge Questionnaire}

Maternal knowledge questionnaire was adapted from [19] and was translated into Arabic language by the researchers. The 40- close-ended items questionnaire designed to measure maternal knowledge regarding antenatal care and consisted of (6) sections. Section (1) general knowledge regarding antenatal care (5 items), section (2) knowledge about lifestyle modifications during pregnancy (7 items), section (3) knowledge about minor discomfort during pregnancy (8 items), section (4) knowledge about danger signs during pregnancy (7 items), section (5) knowledge about diet system during pregnancy (6 items), and section (6) knowledge about the necessary investigations during pregnancy (7 items).

\section{Scoring}

The pregnant women who checked an item (yes) was given (2), while the one who checked an item (No) was given (1) and the one who checked an item (Don't know) was given (0). As well as, women' total knowledge score was classified as the following:

- Poor when the total score was less than $60 \%$

- Average when the total score was $60 \%$ to less than $75 \%$

- Good when total score was $75 \%$ to $100 \%$.

\subsubsection{Third Tool}

Maternal Health Attitude Questionnaire (MHAQ):

The MHAQ was adapted from [10] and was translated into Arabic language by the researchers. The 15 items questionnaire to assess attitude focused on dietary habits changes, supplementation of iron and folic acid, the effect of smoking on mother and fetus, effects of alcohol on the health of mother and fetus, dental care, effect of tight clothes on the health of the fetus, visits, investigations, and the opinion of the woman about the place of delivery.

\section{Scoring:}

The MHAQ has a three point Likert-type scale ranging from 1 (agree), 2 (neutral) and 3 (disagree). An overall score of the MHAQ is a calculation of the mean of responses from all items. As well as women' total attitude score was classified as the following:

- Negative attitude when the total attitude score was less than $60 \%$

- Positive attitude when the total attitude score was 60-100\%.

\subsubsection{Fourth Tool}

- Maternal Health Practices Questionnaire:

It was designed by the researchers after reviewing related literature $[10,20]$. It was written in an Arabic language. It was consisted of 20-items which designed to measure maternal practices regarding antenatal care. Items focused on the regularity of Ante Natal Care visits, avoiding tea and coffee drinks, brushing teeth, avoiding tight clothes, avoiding high heel shoes, bathing in standing position, rest and sleep, getting exercise, choose a diet low in fat, limit use of sugars, increase protein intake, increase green leafy vegetables, increase milk intake, increase fluid intake, taking iron and folic acid tablets, monitor blood pressure regularly and report any unusual signs or symptoms to a physician.

\section{Scoring:}

The items were judged according to three point likert scale continuum from never (1), sometimes (2), and always (3). Then, summing up the scores of the items and the overall scores gave the health practices score. As well as women' total practices score was classified as the following:

- Poor when the total score was less than $60 \%$.

- Fair when the total score was $60 \%$ to less than $75 \%$.

- Good when total score was $75 \%$ to $100 \%$.

\subsubsection{Fifth Tool}

- Women's Satisfaction Sheet:

Women's satisfaction was evaluated by using a visual analog satisfaction scale (VASS). The VAS was adopted from [21]. The VAS scale is an instrument in which 0 (zero) represents that the subject was unsatisfied with the antenatal instructional package implementation and 10 highly satisfied. Scoring:

- 0 = Unsatisfied

- 1-9 = Satisfied

- 10 = Highly satisfied.

\subsection{Method}

The research was executed according to the following steps:

\subsubsection{Administrative Approval}

This research was conducted under the approval of the Faculty of Nursing Ethical Committee, Benha University. An official permission was obtained from the directors of 
the pre-mentioned setting to conduct the study after explaining its purpose.

\subsubsection{Validity}

The tools of data collection were submitted to a panel of three nursing experts in the field of obstetrics and gynaecology to test the content validity, modification were carried out according to the panel' judgments on clarity of sentences and the appropriateness of content.

\subsubsection{Reliability}

The reliability was done by Cronbach's Alpha coefficient test which revealed that each of the five tools consisted of relatively homogenous items as indicated by the moderate to high reliability of each tool was (0.86).

\subsubsection{Ethical Considerations}

- Approvals of women were obtained before data collection and after explaining the purpose of the research.

- Anonymity was assured as the filled questionnaire sheets were given a code number (not by names).

- The women were ensured that questionnaire sheet will be used only for the purpose of the research and will be discarded at the end of the research.

- The research maneuvers do not entail any harmful effects on participation.

- The women who participated in the research were informed about having the right to withdraw at any time without giving any reason.

\subsubsection{A Pilot Study}

The pilot study was carried out on $10 \%$ of total duration of collected data (18 days) to test the simplicity, clarity and applicability of the study tools as well as estimation of the time needed to fill the questionnaire. According to the results of the pilot study, required modifications were done in the form of adding, omission or paraphrasing of some questions. Women involved in the pilot excluded from the study to avoid contamination of the sample.

The purposes of the pilot study were to:

- Ascertain the clarity and the applicability of the tools

- Ascertain the relevance and content validity of the tools.

- $\quad$ Estimate the time needed to complete the sheet.

- Detect any problem peculiar to the statements such as sequence and clarity that might interfere with the process of data collection. The necessary changes were undertaken.

The results of the pilot study:

After conducting the pilot study, it was found that:

- The tools were clear and applicable; however, few words were modified

- Tools were relevant and valid.

- No problem that interferes with the process of data collection was detected.

- Following this pilot study the tools were made ready for use.

\subsubsection{Field Work}

To fulfil the aim of this research, the following phases were adopted, preparatory phase, interviewing and assessment phase, planning phase, implementation of the antenatal instructional package phase and evaluation phase. These phases were carried out from the beginning of August, 2018 and completed at the end of January, 2019 covering six months. The researcher visited the previously mentioned setting three days/week, (Sunday, Tuesday, Thursday), from 9.00 Am to 12.00 Pm.

\section{A. Preparatory phase:}

The preparatory phase was the first phase of the research, the researchers carried out through review of local and international related literature about the various aspects of the research problem. This helped the researchers to be acquainted with magnitude and seriousness of the problem, and guided the researchers to prepare the required data collection tools.

\section{B. Interviewing and assessment phase:}

This phase encompassed interviewing both control and study group. Interviewing began first with control group to avoid bias then with study group. At the beginning of interview the researchers greeted the woman, introduced herself to each woman included in the research, explained the purpose of the research and provided the woman with all information about the research (purpose, duration, and activities) and take oral consent to participate in the research. Data were collected by the researchers through administration of the tools (A structured interviewing questionnaire, maternal knowledge questionnaire, maternal health attitude questionnaire and maternal health practices questionnaire) to each woman. Average time for the completion of each woman interview was around (15-20 minutes). A number of interviewed women / week ranged from (6-8) women.

\section{Planning phase:}

Based on results obtained from study group during assessment phase, the antenatal instructional package was developed by the researchers in a form of printed Arabic booklet to satisfy the studied pregnant women's deficit knowledge, attitude and practices regarding antenatal care during pregnancy. Sessions number and its contents, different methods of teaching, and instructional media were determined accordingly to study group. Objectives of antenatal instructional package were constructed and included the following:

\subsection{General Objectives}

By the end of the antenatal instructional package' sessions, each woman in the study group will be able to acquire essential knowledge, healthy attitude and healthy practices regarding antenatal care and will be satisfied with the instructional package regarding antenatal care.

\subsection{Specific Objectives}

By the end of the antenatal instructional package' sessions, each woman in the study group will be able to:

- Define antenatal care.

- Discuss lifestyle during pregnancy.

- Identify diet system during pregnancy.

- Enumerate minor disturbance of pregnancy.

- Discuss danger signs of pregnancy.

- Identify the frequency of antenatal care visits.

- Identify the necessary investigations during pregnancy. 
- Adopt healthy attitude and practices during pregnancy.

\section{A. Implementation of the antenatal instructional package phase:}

Pregnant women in the control group received only routine care by hospital staff while pregnant women in the study group received routine care by hospital staff, in addition to participated in the antenatal instructional package through three scheduled sessions.

Each session took about 45-60 minutes. At the beginning of the first session women were oriented with the antenatal instructional package' contents. Each woman was informed about the time of the next sessions at the end of the session. The subsequent session started by a feedback about the previous session and the objectives of the new session, simple Arabic language was used to suit women' level of understanding. At the end of each session, women' questions were discussed to correct any misunderstanding.

The first session of the antenatal instructional package implementation began during the women' first visit that follow the interviewing phase and included, definition of antenatal care, lifestyle during pregnancy and diet system during pregnancy, the second session of the antenatal instructional package implementation began during the women' second visit that included, minor disturbance of pregnancy and warning signs of pregnancy and the third session of the antenatal instructional package implementation began during the women' third visit that included, frequency of antenatal care visits, the necessary investigations during pregnancy and healthy practices during pregnancy. These sessions were repeated to each subgroup of (4-6) women.

Different methods of teaching were used such as discussion, demonstration, re-demonstration and brainstorming. Instructional media included videos contain all content of the ANC instructional package and educational booklet about antenatal care which constructed by the researchers in a simple Arabic language after reviewing the related literatures were distributed to all recruited women in the study to achieve its objectives.

\section{B- Evaluation phase:}

The effectiveness of the antenatal instructional package was evaluated one month after antenatal instructional package implementation using the same format of tools which used before implementation for both groups. Evaluation started first with control group then with study group to avoid bias. As well as, the women's satisfaction regarding the antenatal instructional package implementation was evaluated using visual analog satisfaction scale. At almost time the researchers followed women via telephone. Also, according to principles of ethics in research, the members of the control group were provided by the educational booklet that prepared by the researchers after evaluation.

\subsection{Statistical Design}

Data was verified prior to computerized entry. The Statistical Package for Social Sciences (SPSS version 22.0) was used for that purpose, followed by data tabulation and analysis. Descriptive statistics were applied (e.g., mean, standard deviation, frequency and percentages). Test of significance (t test, chi-square). A significant level value was considered when $\mathrm{p} \leq 0.05$. In addition, $A$ highly significant level value was considered when $\mathrm{p}<0.001$.

\subsection{Limitation of the Research}

Sometimes the sessions were protracted due to noise and other individuals' interruption.

\section{Results}

Table 1 reveals that there was no statistically significant difference between study and control group regarding socio demographic characteristics.

Table 2 clarifies that there was no statistically significant difference between study and control group regarding obstetric history.

Figure 1 shows that nearly one third of studied women in both study group (41.4\%) and control group (37.1\%) had source of information from friends and families while minority of them had source of information from newspaper.

Table 3 shows that, there was no statistical significant difference regarding all knowledge items about antenatal care between the study and the control group before instructional package implementation. However, a highly statistically significant difference (p-values < 0.001) was observed regarding all knowledge items about antenatal care between the study and the control group one month after instructional package implementation.

Figure 2 represents that total knowledge score regarding antenatal care were greatly improved one month after instructional package implementation than before implementation among study group while there were minimal improvement in total knowledge score one month after instructional package implementation than before implementation among control group.

Table 4 clarifies that, there was no statistical significant difference regarding all attitude items about antenatal care between the study and the control group before instructional package implementation. However, a highly statistically significant difference ( $p$-values $<0.001$ ) was observed regarding all attitude items about antenatal care between the study and the control group one month after instructional package implementation.

Figure 3 represents that total attitude regarding antenatal care were greatly improved one month after instructional package implementation than before implementation among study group while there were minimal improvements in total attitude one month after instructional package implementation than before implementation among control group.

Table 5 shows that, there was no statistical significant difference regarding all practices item about antenatal care between the study and the control group before instructional package implementation. However, a highly statistically significant difference ( $p$-values $<0.001$ ) was observed regarding all practices item about antenatal care between the study and the control group one month after instructional package implementation. 
Table 1. Distribution of studied groups regarding socio-demographic characteristics (n=140)

\begin{tabular}{|c|c|c|c|c|c|c|}
\hline \multirow[t]{2}{*}{ Socio-demographic characteristics } & \multicolumn{2}{|c|}{$\begin{array}{c}\text { Study group } \\
n=70\end{array}$} & \multicolumn{2}{|c|}{$\begin{array}{c}\begin{array}{c}\text { Control group } \\
n=70\end{array} \\
\end{array}$} & \multirow[t]{2}{*}{$\mathbf{X}^{2}$} & \multirow[t]{2}{*}{ p-value } \\
\hline & No & $\%$ & No & $\%$ & & \\
\hline \multicolumn{7}{|l|}{ Age (years) } \\
\hline$<25$ years & 24 & 34.3 & 30 & 42.9 & \multirow{4}{*}{1.18} & \multirow{4}{*}{$<0.05$} \\
\hline $25-<30$ years & 38 & 54.3 & 32 & 45.7 & & \\
\hline $30-<35$ years & 8 & 11.4 & 8 & 11.4 & & \\
\hline Mean \pm SD & \multicolumn{2}{|c|}{$24.81 \pm 4.54$} & \multicolumn{2}{|c|}{$25.85 \pm 4.11$} & & \\
\hline \multicolumn{7}{|l|}{ Residence } \\
\hline Rural & 47 & 67.1 & 41 & 58.6 & \multirow{2}{*}{1.10} & \multirow{2}{*}{$<0.05$} \\
\hline Urban & 23 & 32.9 & 29 & 41.4 & & \\
\hline \multicolumn{7}{|l|}{ Educational level } \\
\hline Primary education & 7 & 10.0 & 10 & 14.3 & \multirow{4}{*}{1.88} & \multirow{4}{*}{$<0.05$} \\
\hline Preparatory education & 7 & 10.0 & 11 & 15.7 & & \\
\hline Secondary education & 32 & 45.7 & 28 & 40.0 & & \\
\hline University education & 24 & 34.3 & 21 & 30.0 & & \\
\hline \multicolumn{7}{|l|}{ Occupation } \\
\hline Housewife & 17 & 24.3 & 17 & 24.3 & \multirow{3}{*}{0.77} & \multirow{3}{*}{$<0.05$} \\
\hline Employed & 41 & 58.6 & 37 & 52.9 & & \\
\hline Students & 12 & 17.1 & 16 & 22.9 & & \\
\hline \multicolumn{7}{|l|}{ Income } \\
\hline Enough & 58 & 82.9 & 61 & 87.1 & \multirow[b]{2}{*}{0.50} & \multirow[b]{2}{*}{$<0.05$} \\
\hline Not enough & 12 & 17.1 & 9 & 12.9 & & \\
\hline \multicolumn{7}{|l|}{ Transportation } \\
\hline Motorcycle & 18 & 25.7 & 20 & 28.6 & \multirow{3}{*}{0.76} & \multirow{3}{*}{$<0.05$} \\
\hline Car & 29 & 41.4 & 24 & 34.3 & & \\
\hline Walking & 23 & 32.9 & 26 & 37.1 & & \\
\hline
\end{tabular}

Table 2. Distribution of studied groups regarding obstetric history $(n=140)$

\begin{tabular}{|c|c|c|c|c|c|c|}
\hline \multirow[t]{2}{*}{ Obstetric history } & \multicolumn{2}{|c|}{$\begin{array}{c}\text { Study group } \\
\mathbf{n}=70\end{array}$} & \multicolumn{2}{|c|}{$\begin{array}{c}\text { Control group } \\
\mathbf{n}=70\end{array}$} & \multirow[t]{2}{*}{$\mathbf{X}^{2}$} & \multirow[t]{2}{*}{ p-value } \\
\hline & No & $\%$ & No & $\%$ & & \\
\hline \multicolumn{7}{|c|}{ Number of pregnancy } \\
\hline One & 35 & 50.0 & 31 & 44.3 & \multirow{3}{*}{0.81} & \multirow{3}{*}{$<0.05$} \\
\hline Two & 23 & 32.9 & 23 & 32.9 & & \\
\hline Three or more & 12 & 17.1 & 16 & 22.9 & & \\
\hline Within 4 weeks & 6 & 8.6 & 7 & 10.0 & \multirow{3}{*}{0.74} & \multirow{3}{*}{$<0.05$} \\
\hline 4weeks-8weeks & 44 & 62.9 & 39 & 55.7 & & \\
\hline 8weeks-12weeks & 20 & 28.6 & 24 & 34.3 & & \\
\hline
\end{tabular}

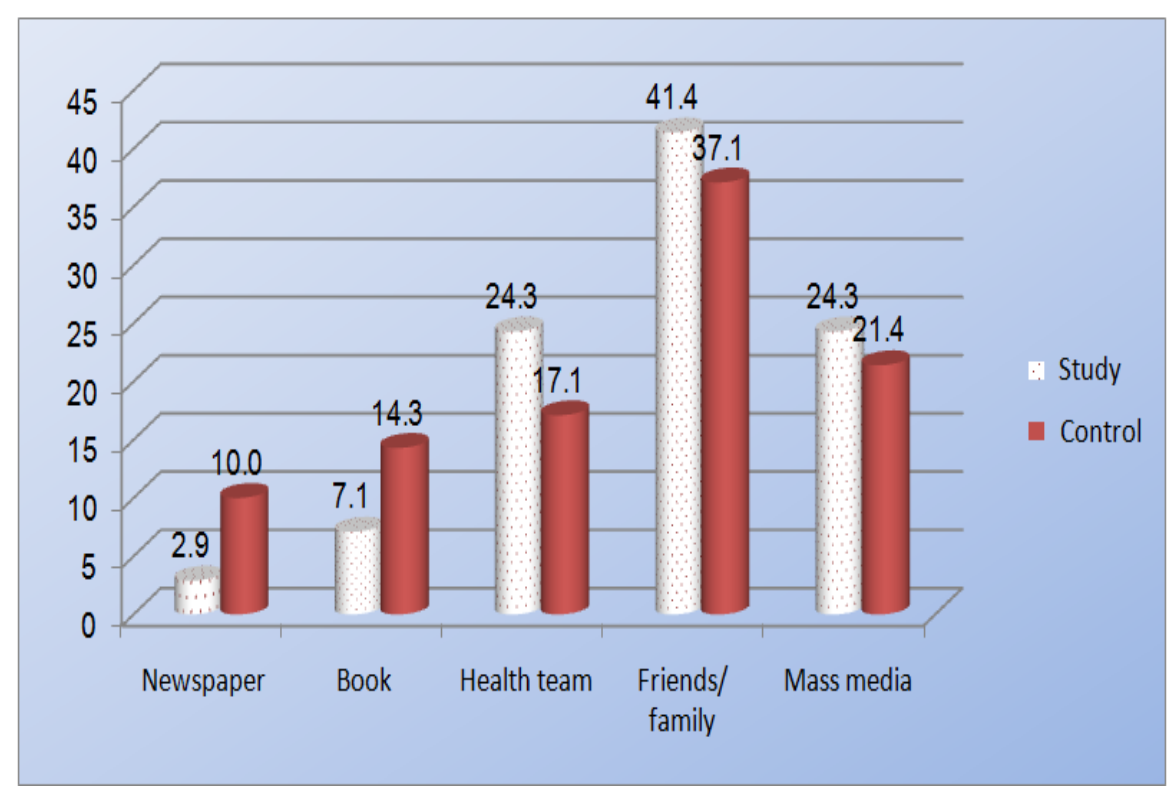

Figure 1. Frequency distribution of studied groups regarding source of information about ANC 
Table 3. Distribution of studied groups according to all knowledge items about antenatal care before and one month after instructional package implementation $(n=140)$

\begin{tabular}{|c|c|c|c|c|c|c|c|c|c|c|c|c|}
\hline \multirow{3}{*}{$\begin{array}{l}\text { Knowledge } \\
\text { items }\end{array}$} & \multicolumn{4}{|c|}{$\begin{array}{c}\text { Before instructional package } \\
\text { implementation }\end{array}$} & \multirow{3}{*}{$\mathrm{X}^{2}$} & \multirow{3}{*}{ p-value } & \multicolumn{4}{|c|}{$\begin{array}{c}\text { One month after instructional package } \\
\text { implementation }\end{array}$} & \multirow{3}{*}{$\mathrm{X}^{2}$} & \multirow{3}{*}{ p-value } \\
\hline & \multicolumn{2}{|c|}{$\begin{array}{c}\text { Study group } \\
\mathbf{n}=\mathbf{7 0}\end{array}$} & \multicolumn{2}{|c|}{$\begin{array}{c}\text { Control group } \\
\mathbf{n}=70\end{array}$} & & & \multicolumn{2}{|c|}{$\begin{array}{c}\text { Study group } \\
n=70\end{array}$} & \multicolumn{2}{|c|}{$\begin{array}{c}\text { Control group } \\
\mathbf{n}=70\end{array}$} & & \\
\hline & No & $\%$ & No & $\%$ & & & No & $\%$ & no & $\%$ & & \\
\hline \multicolumn{13}{|c|}{ 1-knowledge about antenatal care visits } \\
\hline I don't know & 18 & 25.7 & 16 & 22.9 & \multirow{3}{*}{0.18} & \multirow{3}{*}{0.91} & 7 & 10.0 & 16 & 22.9 & \multirow{3}{*}{11.8} & \multirow{3}{*}{$0.003^{*}$} \\
\hline No & 22 & 31.4 & 22 & 31.4 & & & 6 & 8.6 & 16 & 22.9 & & \\
\hline Yes & 30 & 42.9 & 32 & 45.7 & & & 57 & 81.4 & 38 & 54.3 & & \\
\hline \multicolumn{13}{|c|}{ 2-knowledge about lifestyle during pregnancy } \\
\hline I don't know & 11 & 15.7 & 13 & 18.6 & \multirow{3}{*}{0.48} & \multirow{3}{*}{0.79} & 3 & 4.3 & 9 & 12.9 & \multirow{3}{*}{21.0} & \multirow{3}{*}{$0.000^{*}$} \\
\hline No & 23 & 32.9 & 25 & 35.7 & & & 6 & 8.6 & 25 & 35.7 & & \\
\hline Yes & 36 & 51.4 & 32 & 45.7 & & & 61 & 87.1 & 36 & 51.4 & & \\
\hline \multicolumn{13}{|c|}{ 3-knowledge about minor discomfort during pregnancy } \\
\hline I don't know & 27 & 38.6 & 23 & 32.9 & \multirow{3}{*}{3.31} & \multirow{3}{*}{0.19} & 8 & 11.4 & 23 & 32.9 & \multirow{3}{*}{15.0} & \multirow{3}{*}{$0.001^{*}$} \\
\hline No & 18 & 25.7 & 28 & 40.0 & & & 20 & 28.6 & 26 & 37.1 & & \\
\hline Yes & 25 & 35.7 & 19 & 27.1 & & & 42 & 60.0 & 21 & 30.0 & & \\
\hline \multicolumn{13}{|c|}{ 4-knowledge about danger signs during pregnancy } \\
\hline I don't know & 35 & 50.0 & 25 & 35.7 & \multirow{3}{*}{3.29} & \multirow{3}{*}{0.19} & 7 & 10.0 & 23 & 32.9 & & \\
\hline No & 18 & 25.7 & 20 & 28.6 & & & 13 & 18.6 & 18 & 25.7 & 14.9 & $0.001^{*}$ \\
\hline Yes & 17 & 24.3 & 25 & 35.7 & & & 50 & 71.4 & 29 & 41.4 & & \\
\hline 5-knowledge al & diet $s$ & m dur & preg & & & & & & & & & \\
\hline I don't know & 24 & 34.3 & 22 & 31.4 & & & 7 & 10.0 & 21 & 30.0 & & \\
\hline No & 27 & 38.6 & 26 & 37.1 & 0.32 & 0.85 & 13 & 18.6 & 22 & 31.4 & 16.1 & $0.000 *$ \\
\hline Yes & 19 & 27.1 & 22 & 31.4 & & & 50 & 71.4 & 27 & 38.6 & & \\
\hline 6-knowledge al & the $n$ & sary i & stigat & during & gnanc & & & & & & & \\
\hline I don't know & 52 & 74.3 & 50 & 71.4 & & & 14 & 20.0 & 38 & 54.3 & & \\
\hline No & 9 & 12.9 & 10 & 14.3 & 0.14 & 0.93 & 17 & 24.3 & 10 & 14.3 & 17.6 & $0.000 *$ \\
\hline Yes & 9 & 12.9 & 10 & 14.3 & & & 39 & 55.7 & 22 & 31.4 & & \\
\hline
\end{tabular}

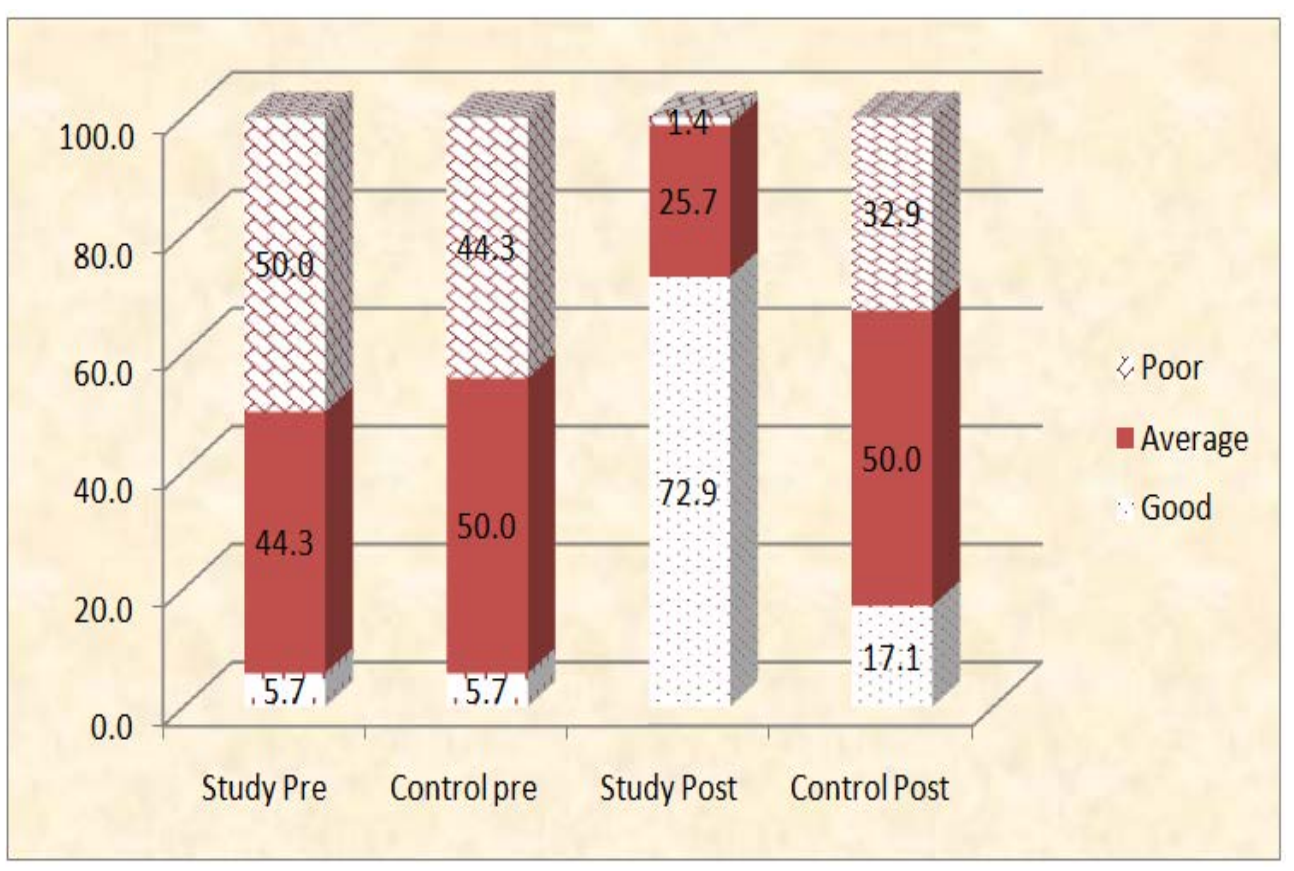

Figure 2. Frequency distribution of studied groups regarding total knowledge before and one month after instructional package implementation 
Table 4. Distribution of studied groups according to attitude regarding antenatal care before and one month after instructional package implementation $(\mathrm{n}=140)$

\begin{tabular}{|c|c|c|c|c|c|c|c|c|c|c|c|c|}
\hline \multirow{3}{*}{ Attitude items } & \multicolumn{4}{|c|}{$\begin{array}{c}\text { Before instructional package } \\
\text { implementation }\end{array}$} & \multirow{3}{*}{$X^{2}$} & \multirow{3}{*}{ p-value } & \multicolumn{4}{|c|}{$\begin{array}{c}\text { One month after instructional package } \\
\text { implementation }\end{array}$} & \multirow{3}{*}{$X^{2}$} & \multirow{3}{*}{ p-value } \\
\hline & \multicolumn{2}{|c|}{$\begin{array}{l}\text { Study group } \\
\quad \mathrm{n}=70\end{array}$} & \multicolumn{2}{|c|}{$\begin{array}{c}\text { Control group } \\
\mathbf{n}=70\end{array}$} & & & \multicolumn{2}{|c|}{$\begin{array}{c}\text { Study group } \\
\mathbf{n}=70\end{array}$} & \multicolumn{2}{|c|}{$\begin{array}{c}\text { Control group } \\
n=70\end{array}$} & & \\
\hline & No & $\%$ & No & $\%$ & & & No & $\%$ & No & $\%$ & & \\
\hline 1-Early antena & $\lg$ is & or pre & ncy & & & & & & & & & \\
\hline Disagree & 20 & 28.6 & 17 & 24.3 & & & 2 & 2.9 & 15 & 21.4 & & \\
\hline Neutral & 22 & 31.4 & 23 & 32.9 & 033 & 084 & 12 & 17.1 & 21 & 30.0 & 177 & $0000 *$ \\
\hline Agree & 28 & 40.0 & 30 & 42.9 & 0.33 & 0.84 & 56 & 80.0 & 34 & 48.6 & 17.1 & $0.000^{\uparrow}$ \\
\hline 2-Antenatal fol & good & onitor & her's & fetus' l & alth & & & & & & & \\
\hline Disagree & 11 & 15.7 & 11 & 15.7 & & & 2 & 2.9 & 9 & 12.9 & & \\
\hline Neutral & 23 & 32.9 & 25 & 35.7 & 014 & 093 & 12 & 17.1 & 24 & 34.3 & 123 & $0002 *$ \\
\hline Agree & 36 & 51.4 & 34 & 48.6 & 0.14 & 0.93 & 56 & 80.0 & 37 & 52.9 & 12.3 & $0.002^{*}$ \\
\hline 3-Pregnant woI & I ch & dietary & bit as : & sed by & ctor & & & & & & & \\
\hline Disagree & 16 & 22.9 & 18 & 25.7 & & & 4 & 5.7 & 16 & 22.9 & & \\
\hline Neutral & 33 & 47.1 & 29 & 41.4 & 046 & 097 & 7 & 10.0 & 28 & 40.0 & 326 & $0000 *$ \\
\hline Agree & 21 & 30.0 & 23 & 32.9 & 0.46 & 0.97 & 59 & 84.3 & 26 & 37.1 & 32.6 & $0.000^{x}$ \\
\hline 4-Supplementa & n an & c acid & $\operatorname{good} f$ & he motl & $r$ and & & & & & & & \\
\hline Disagree & 12 & 17.1 & 14 & 20.0 & & & 3 & 4.3 & 14 & 20.0 & & \\
\hline Neutral & 18 & 25.7 & 19 & 27.1 & ק 0 0 & 096 & 3 & 4.3 & 19 & 27.1 & 250 & $00 \Omega 0 *$ \\
\hline Agree & 40 & 57.1 & 37 & 52.9 & 0.29 & 0.86 & 64 & 91.4 & 37 & 52.9 & 25.9 & $0.000^{\uparrow}$ \\
\hline 5-Smoking is $\mathrm{h}$ & the $f$ & & & & & & & & & & & \\
\hline Disagree & 17 & 24.3 & 21 & 30.0 & & & 3 & 4.3 & 18 & 25.7 & & \\
\hline Neutral & 18 & 25.7 & 17 & 24.3 & 0.58 & 0.74 & 6 & 8.6 & 14 & 20.0 & 10? & م \\
\hline Agree & 35 & 50.0 & 32 & 45.7 & & & 61 & 87.1 & 38 & 54.3 & 19.2 & $0.000^{*}$ \\
\hline 6-Alcohol const & Iurin & gnancy & 11 affec & e fetal $q$ & owth & & & & & & & \\
\hline Disagree & 9 & 12.9 & 6 & 8.6 & & & 3 & 4.3 & 6 & 8.6 & & \\
\hline Neutral & 23 & 32.9 & 25 & 35.7 & 0.69 & 0.70 & 8 & 11.4 & 22 & 31.4 & 103 & $0006 *$ \\
\hline Agree & 38 & 54.3 & 39 & 55.7 & & & 59 & 84.3 & 42 & 60.0 & 10.3 & $0.006^{\top}$ \\
\hline 7-Health educa & gien & ctices i & porta & uring a & enatal & isits & & & & & & \\
\hline Disagree & 18 & 25.7 & 15 & 21.4 & & & 2 & 2.9 & 15 & 21.4 & & \\
\hline Neutral & 18 & 25.7 & 27 & 38.6 & 2.65 & 0.26 & 9 & 12.9 & 26 & 37.1 & 28.4 & $0000 *$ \\
\hline Agree & 34 & 48.6 & 28 & 40.0 & & & 59 & 84.3 & 29 & 41.4 & & \\
\hline 8-Brushing of $t$ & dail & ecessa & uring & gnancy & & & & & & & & \\
\hline Disagree & 27 & 38.6 & 21 & 30.0 & & & 6 & 8.6 & 17 & 24.3 & & \\
\hline Neutral & 30 & 42.9 & 38 & 54.3 & 1.85 & 0.39 & 3 & 4.3 & 33 & 47.1 & 510 & $0000 *$ \\
\hline Agree & 13 & 18.6 & 11 & 15.7 & & & 61 & 87.1 & 20 & 28.6 & 51.0 & $0.000^{*}$ \\
\hline 9-Tight clothes & regne & ffect $t$ & etus' h & & & & & & & & & \\
\hline Disagree & 21 & 30.0 & 15 & 21.4 & & & 5 & 7.1 & 13 & 18.6 & & \\
\hline Neutral & 23 & 32.9 & 23 & 32.9 & 1.62 & 0.44 & 0 & 0.0 & 18 & 25.7 & 280 & $0000 *$ \\
\hline Agree & 26 & 37.1 & 32 & 45.7 & & & 65 & 92.9 & 39 & 55.7 & 28.0 & $0.000^{*}$ \\
\hline 10-Screening o & rinfe & s(HIV & BV) sh & d be car & ied out & luring an & natal ch & & & & & \\
\hline Disagree & 14 & 20.0 & 15 & 21.4 & & & 7 & 10.0 & 15 & 21.4 & & \\
\hline Neutral & 41 & 58.6 & 27 & 38.6 & 6.84 & $0.03 *$ & 12 & 17.1 & 26 & 37.1 & 141 & $0001 *$ \\
\hline Agree & 15 & 21.4 & 28 & 40.0 & & & 51 & 72.9 & 29 & 41.4 & 14.1 & $0.001^{*}$ \\
\hline 11-Blood suga & uld b & ried ou & Iring a & natal ch & ck-up & & & & & & & \\
\hline Disagree & 11 & 15.7 & 6 & 8.6 & & & 6 & 8.6 & 5 & 7.1 & & \\
\hline Neutral & 30 & 42.9 & 28 & 40.0 & 2.29 & 0.31 & 3 & 4.3 & 26 & 37.1 & 2 & $0000 *$ \\
\hline Agree & 29 & 41.4 & 36 & 51.4 & & & 61 & 87.1 & 39 & 55.7 & 23.1 & $0.000^{*}$ \\
\hline 12-Urine test fo & a inf & is nec & ary du & pregna & & & & & & & & \\
\hline Disagree & 17 & 24.3 & 17 & 24.3 & & & 3 & 4.3 & 11 & 15.7 & & \\
\hline Neutral & 23 & 32.9 & 25 & 35.7 & 0.15 & 0.92 & 1 & 1.4 & 19 & 27.1 & 27 & م \\
\hline Agree & 30 & 42.9 & 28 & 40.0 & & & 66 & 94.3 & 40 & 57.2 & 27.1 & $0.000^{*}$ \\
\hline 13-Blood press & be & ed reg & ly dur & pregna & & & & & & & & \\
\hline Disagree & 16 & 22.9 & 17 & 24.3 & & & 3 & 4.3 & 14 & 20.0 & & \\
\hline Neutral & 26 & 37.1 & 18 & 25.7 & 2.26 & 0.32 & 5 & 7.1 & 18 & 25.7 & 20 & $0000 *$ \\
\hline Agree & 28 & 40.0 & 35 & 50.0 & & & 62 & 88.6 & 38 & 54.3 & & \\
\hline 14-Pregnant $w$ & Ild u & o ultr: & ind sca & s advise & by do & or to mo & or fetal & & & & & \\
\hline Disagree & 25 & 35.7 & 30 & 42.9 & & & 6 & 8.6 & 30 & 42.9 & & \\
\hline Neutral & 27 & 38.6 & 19 & 27.1 & 2.07 & 0.35 & 3 & 4.3 & 19 & 27.1 & 4714 & $0.000 *$ \\
\hline Agree & 18 & 25.7 & 21 & 30.0 & & & 61 & 87.1 & 21 & 30.0 & 47.14 & $0.000^{*}$ \\
\hline 15-Hospital del & etter & home & very & & & & & & & & & \\
\hline Disagree & 15 & 21.4 & 15 & 21.4 & & & 2 & 2.9 & 15 & 21.4 & & \\
\hline Neutral & 22 & 31.4 & 26 & 37.1 & 0.59 & 0.74 & 2 & 2.9 & 26 & 37.1 & 449 & $0000 *$ \\
\hline Agree & 33 & 47.1 & 29 & 41.4 & & & 66 & 94.3 & 29 & 41.4 & 44.9 & $0.000{ }^{*}$ \\
\hline
\end{tabular}




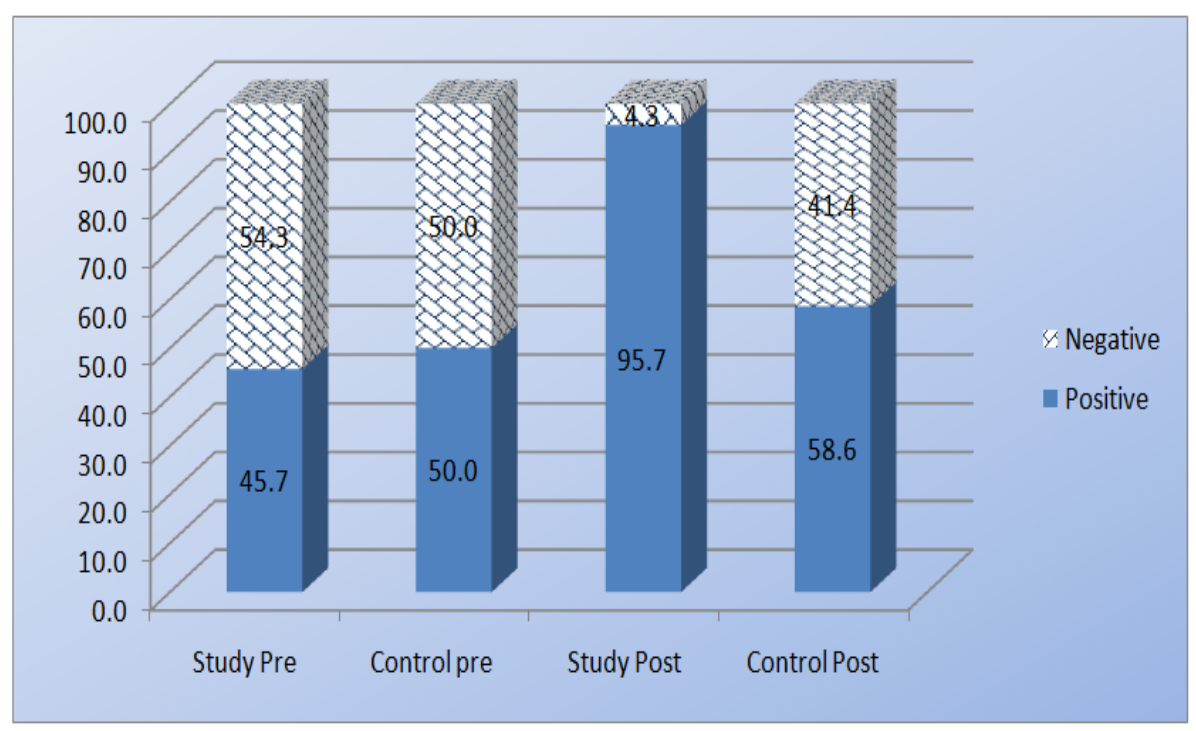

Figure 3. Frequency distribution of studied groups regarding total attitude before and one month after instructional package implementation

Table 5. Distribution of studied groups according to their total practices regarding antenatal care before and one month after instructional package implementation $(\mathbf{n}=\mathbf{1 4 0})$

\begin{tabular}{|c|c|c|c|c|c|c|c|c|c|c|c|c|}
\hline \multirow{3}{*}{ Practices items } & \multicolumn{4}{|c|}{$\begin{array}{c}\text { Before instructional package } \\
\text { implementation }\end{array}$} & \multirow{3}{*}{$\mathrm{X}^{2}$} & \multirow{3}{*}{ p-value } & \multicolumn{4}{|c|}{$\begin{array}{c}\text { One month after instructional package } \\
\text { implementation }\end{array}$} & \multirow{3}{*}{$\mathbf{X}^{2}$} & \multirow{3}{*}{ p-value } \\
\hline & \multicolumn{2}{|c|}{$\begin{array}{c}\text { Study group } \\
n=70\end{array}$} & \multicolumn{2}{|c|}{$\begin{array}{c}\text { Control group } \\
n=70\end{array}$} & & & \multicolumn{2}{|c|}{$\begin{array}{c}\text { Study group } \\
\mathbf{n}=\mathbf{7 0}\end{array}$} & \multicolumn{2}{|c|}{$\begin{array}{c}\text { Control group } \\
n=70\end{array}$} & & \\
\hline & No & $\%$ & No & $\%$ & & & No & $\%$ & No & $\%$ & & \\
\hline \multicolumn{13}{|c|}{ 1-Regular in Ante Natal Care visits (ANC-Visits) for check-up as doctor order } \\
\hline Never & 21 & 30.0 & 25 & 35.7 & \multirow{3}{*}{1.53} & \multirow{3}{*}{0.46} & 2 & 2.9 & 10 & 14.3 & \multirow{3}{*}{19.1} & \multirow{3}{*}{$0.000 *$} \\
\hline Sometimes & 19 & 27.1 & 13 & 18.6 & & & 1 & 1.4 & 13 & 18.6 & & \\
\hline Always & 30 & 42.9 & 32 & 45.7 & & & 67 & 95.7 & 47 & 67.1 & & \\
\hline \multicolumn{13}{|c|}{ 2-Avoid drinking tea and coffee during pregnancy } \\
\hline Never & 29 & 41.4 & 29 & 41.4 & \multirow{3}{*}{0.24} & \multirow{3}{*}{0.88} & 2 & 2.9 & 25 & 35.7 & \multirow{3}{*}{53.4} & \multirow{3}{*}{$0.000 *$} \\
\hline Sometimes & 31 & 44.3 & 29 & 41.4 & & & 4 & 5.7 & 23 & 32.9 & & \\
\hline Always & 10 & 14.3 & 12 & 17.1 & & & 64 & 91.4 & 22 & 31.4 & & \\
\hline \multicolumn{13}{|c|}{ 3-Brushing teeth at least twice daily } \\
\hline Never & 21 & 30.0 & 22 & 31.4 & \multirow{3}{*}{0.03} & \multirow{3}{*}{0.98} & 0 & 0 & 15 & 21.4 & \multirow{3}{*}{33.4} & \\
\hline Sometimes & 16 & 22.9 & 16 & 22.9 & & & 0 & 0 & 12 & 17.1 & & $0.000 *$ \\
\hline Always & 33 & 47.1 & 32 & 45.7 & & & 70 & 100.0 & 43 & 61.4 & & \\
\hline 4-Avoiding tight & durin & gnanc & & & & & & & & & & \\
\hline Never & 15 & 21.4 & 15 & 21.4 & & & 2 & 2.9 & 13 & 18.6 & & \\
\hline Sometimes & 34 & 48.6 & 31 & 44.3 & 0.33 & 0.84 & 3 & 4.3 & 29 & 41.4 & 43.9 & $0.000^{*}$ \\
\hline Always & 21 & 30.0 & 24 & 34.3 & & & 65 & 92.9 & 28 & 40.0 & & \\
\hline 5-Avoiding high & es du & pregna & & & & & & & & & & \\
\hline Never & 20 & 28.6 & 17 & 24.3 & & & 10 & 14.3 & 14 & 20.0 & & \\
\hline Sometimes & 31 & 44.3 & 33 & 47.1 & 0.33 & 0.84 & 1 & 1.4 & 31 & 44.3 & 42.5 & $0.000 *$ \\
\hline Always & 19 & 27.1 & 20 & 28.6 & & & 59 & 84.3 & 25 & 35.7 & & \\
\hline 6-Bathing in sta & sition & er that & batl & fear 0 & iscend & Ig infectio & & & & & & \\
\hline Never & 26 & 37.1 & 26 & 37.1 & & & 17 & 24.3 & 24 & 34.3 & & \\
\hline Sometimes & 29 & 41.4 & 24 & 34.3 & 1.18 & 0.55 & 1 & 1.4 & 23 & 32.9 & 32.5 & $0.000 *$ \\
\hline Always & 15 & 21.4 & 20 & 28.6 & & & 52 & 74.3 & 23 & 32.9 & & \\
\hline 7-Sleep 8hr at n & $2 \mathrm{hr}$ i & aftern & & & & & & & & & & \\
\hline Never & 32 & 45.7 & 33 & 47.1 & & & 16 & 22.9 & 22 & 31.4 & & \\
\hline Sometimes & 31 & 44.3 & 28 & 40.0 & 0.41 & 0.81 & 0 & 0 & 32 & 45.7 & 53.5 & $0.000 *$ \\
\hline Always & 7 & 10.0 & 9 & 12.9 & & & 54 & 77.1 & 16 & 22.9 & & \\
\hline 8-Get exercise d & ual de & ctivitie & ich as & king d & ng lun & h, using $s$ & Irs inste & elevato & and $w$ & & & \\
\hline Never & 0 & 0.0 & 6 & 8.6 & & & 12 & 17.1 & 5 & 7.1 & & \\
\hline Sometimes & 49 & 70.0 & 45 & 64.3 & 6.27 & $0.04^{*}$ & 0 & 0.0 & 39 & 55.7 & 54.0 & $0.000 *$ \\
\hline Always & 21 & 30.0 & 19 & 27.1 & & & 58 & 82.9 & 26 & 37.1 & & \\
\hline
\end{tabular}




\begin{tabular}{|c|c|c|c|c|c|c|c|c|c|c|c|c|}
\hline \multirow{3}{*}{ Practices items } & \multicolumn{4}{|c|}{$\begin{array}{c}\text { Before instructional package } \\
\text { implementation }\end{array}$} & \multirow{3}{*}{$\mathrm{X}^{2}$} & \multirow{3}{*}{ p-value } & \multicolumn{4}{|c|}{$\begin{array}{c}\text { One month after instructional package } \\
\text { implementation }\end{array}$} & \multirow{3}{*}{$\mathbf{X}^{2}$} & \multirow{3}{*}{ p-value } \\
\hline & \multicolumn{2}{|c|}{$\begin{array}{c}\text { Study group } \\
n=70\end{array}$} & \multicolumn{2}{|c|}{$\begin{array}{c}\text { Control group } \\
\mathbf{n}=70\end{array}$} & & & \multicolumn{2}{|c|}{$\begin{array}{c}\text { Study group } \\
n=70\end{array}$} & \multicolumn{2}{|c|}{$\begin{array}{c}\text { Control group } \\
n=70\end{array}$} & & \\
\hline & No & $\%$ & No & $\%$ & & & No & $\%$ & No & $\%$ & & \\
\hline \multicolumn{13}{|c|}{ 9-Taking some time for relaxation each day } \\
\hline Never & 19 & 27.1 & 18 & 25.7 & \multirow{3}{*}{0.05} & \multirow{3}{*}{0.97} & 10 & 14.3 & 15 & 21.4 & \multirow{3}{*}{31.2} & \multirow{3}{*}{$0.000 *$} \\
\hline Sometimes & 32 & 45.7 & 32 & 45.7 & & & 2 & 2.9 & 26 & 37.1 & & \\
\hline Always & 19 & 27.1 & 20 & 28.6 & & & 58 & 82.9 & 29 & 41.4 & & \\
\hline \multicolumn{13}{|c|}{ 10-Choose a diet low in fat, saturated fat, and cholesterol. } \\
\hline Never & 26 & 37.1 & 26 & 37.1 & & & 7 & 10.0 & 20 & 28.6 & & \\
\hline Sometimes & 29 & 41.4 & 24 & 34.3 & 1.18 & 0.55 & 0 & 0.00 & 16 & 22.9 & 309 & $0000 *$ \\
\hline Always & 15 & 21.4 & 20 & 28.6 & & & 63 & 90.0 & 34 & 48.6 & & \\
\hline 11-Limiting use & $s$ and & contai & suga & veets). & & & & & & & & \\
\hline Never & 14 & 20.0 & 12 & 17.1 & & & 10 & 14.3 & 11 & 15.7 & & \\
\hline Sometimes & 25 & 35.7 & 24 & 34.3 & 0.31 & 0.85 & 0 & 0 & 24 & 34.3 & 30.6 & $0.000 *$ \\
\hline Always & 31 & 44.3 & 34 & 48.6 & & & 60 & 85.7 & 35 & 50.0 & & \\
\hline 12-Increasing pr & ake e & ally of & nal or & & & & & & & & & \\
\hline Never & 20 & 28.6 & 17 & 24.3 & & & 6 & 8.6 & 17 & 24.3 & & \\
\hline Sometimes & 31 & 44.3 & 33 & 47.1 & 0.33 & 0.84 & 6 & 8.6 & 27 & 38.6 & 30.8 & $0.000 *$ \\
\hline Always & 19 & 27.1 & 20 & 28.6 & & & 58 & 82.9 & 26 & 37.1 & & \\
\hline 13-Increasing gr & vege & $\mathrm{s}$ in $\mathbf{m}$ & & & & & & & & & & \\
\hline Never & 37 & 52.9 & 40 & 57.1 & & & 12 & 17.1 & 38 & 54.3 & & \\
\hline Sometimes & 24 & 34.3 & 20 & 28.6 & 0.53 & 0.76 & 0 & 0.00 & 10 & 14.3 & 39.7 & $0.000 *$ \\
\hline Always & 9 & 12.9 & 10 & 14.3 & & & 58 & 82.9 & 22 & 31.4 & & \\
\hline 14-Increasing m & e dur & e day & & & & & & & & & & \\
\hline Never & 7 & 10.0 & 7 & 10.0 & & & 10 & 14.3 & 13 & 18.6 & & \\
\hline Sometimes & 40 & 57.1 & 43 & 61.4 & 0.31 & 0.85 & 8 & 11.4 & 33 & 47.1 & 259 & $0000 *$ \\
\hline Always & 23 & 32.9 & 20 & 28.6 & & & 52 & 74.3 & 24 & 34.3 & & \\
\hline 15-Taking iron & acid & s duri & regna & as pres & ibed & & & & & & & \\
\hline Never & 7 & 10.0 & 7 & 10.0 & & & 5 & 7.1 & 11 & 15.7 & & \\
\hline Sometimes & 36 & 51.4 & 34 & 48.6 & 0.12 & 0.93 & 8 & 11.4 & 27 & 38.6 & 19.5 & $0.000 *$ \\
\hline Always & 27 & 38.6 & 29 & 41.4 & & & 57 & 81.4 & 32 & 45.7 & & \\
\hline 16-Increasing fl & e $(2-3$ & Iring $t$ & & & & & & & & & & \\
\hline Never & 25 & 35.7 & 25 & 35.7 & & & 7 & 10.0 & 23 & 32.9 & & \\
\hline Sometimes & 31 & 44.3 & 30 & 42.9 & 0.05 & 0.97 & 1 & 1.4 & 18 & 25.7 & 35.7 & $0.000 *$ \\
\hline Always & 14 & 20.0 & 15 & 21.4 & & & 62 & 88.6 & 29 & 41.4 & & \\
\hline 17-Monitoring b & ssure & larly & & & & & & & & & & \\
\hline Never & 29 & 41.4 & 33 & 47.1 & & & 15 & 21.4 & 28 & 40.0 & & \\
\hline Sometimes & 31 & 44.3 & 27 & 38.6 & 0.53 & 0.76 & 6 & 8.6 & 18 & 25.7 & 18.4 & $0.000^{*}$ \\
\hline Always & 10 & 14.3 & 10 & 14.3 & & & 49 & 70.0 & 24 & 34.3 & & \\
\hline 18-Doing the rec & sts du & antena & check & as doct & order & & & & & & & \\
\hline Never & 15 & 21.4 & 15 & 21.4 & & & 6 & 8.6 & 17 & 24.3 & & \\
\hline Sometimes & 33 & 47.2 & 31 & 44.3 & 0.14 & 0.92 & 10 & 14.3 & 24 & 34.3 & 18.5 & $0.000 *$ \\
\hline Always & 22 & 31.4 & 24 & 34.3 & & & 54 & 77.1 & 29 & 41.4 & & \\
\hline 19-Avoiding tak & catio & lout cc & lting & loctor & & & & & & & & \\
\hline Never & 13 & 18.6 & 11 & 15.7 & & & 19 & 27.1 & 13 & 18.6 & & \\
\hline Sometimes & 40 & 57.1 & 40 & 57.1 & 0.27 & 0.87 & 0 & 0.00 & 37 & 52.9 & 51.6 & $0.000^{*}$ \\
\hline Always & 17 & 24.3 & 19 & 27.1 & & & 51 & 72.9 & 20 & 28.6 & & \\
\hline 20-Report any u & igns 0 & iptoms & a phy & & & & & & & & & \\
\hline Never & 12 & 17.1 & 10 & 14.3 & & & 5 & 7.1 & 12 & 17.1 & & \\
\hline Sometimes & 32 & 45.7 & 44 & 62.9 & 4.45 & 0.10 & 2 & 2.9 & 39 & 55.7 & 59.8 & $0.000 *$ \\
\hline Always & 26 & 37.1 & 16 & 22.9 & & & 63 & 90.0 & 19 & 27.1 & & \\
\hline
\end{tabular}




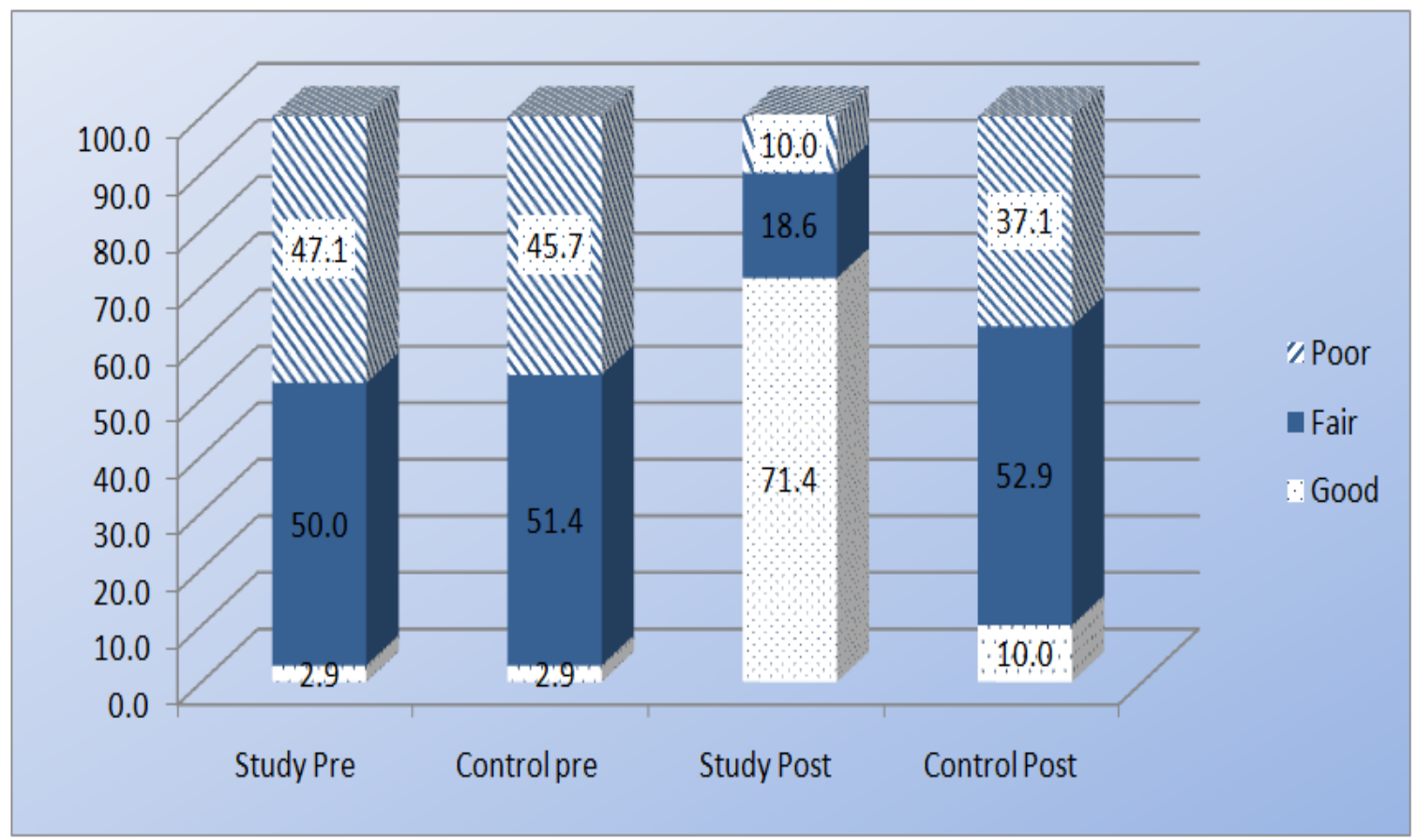

Figure 4. Frequency distribution of studied groups regarding total practices before and one month after instructional package implementation

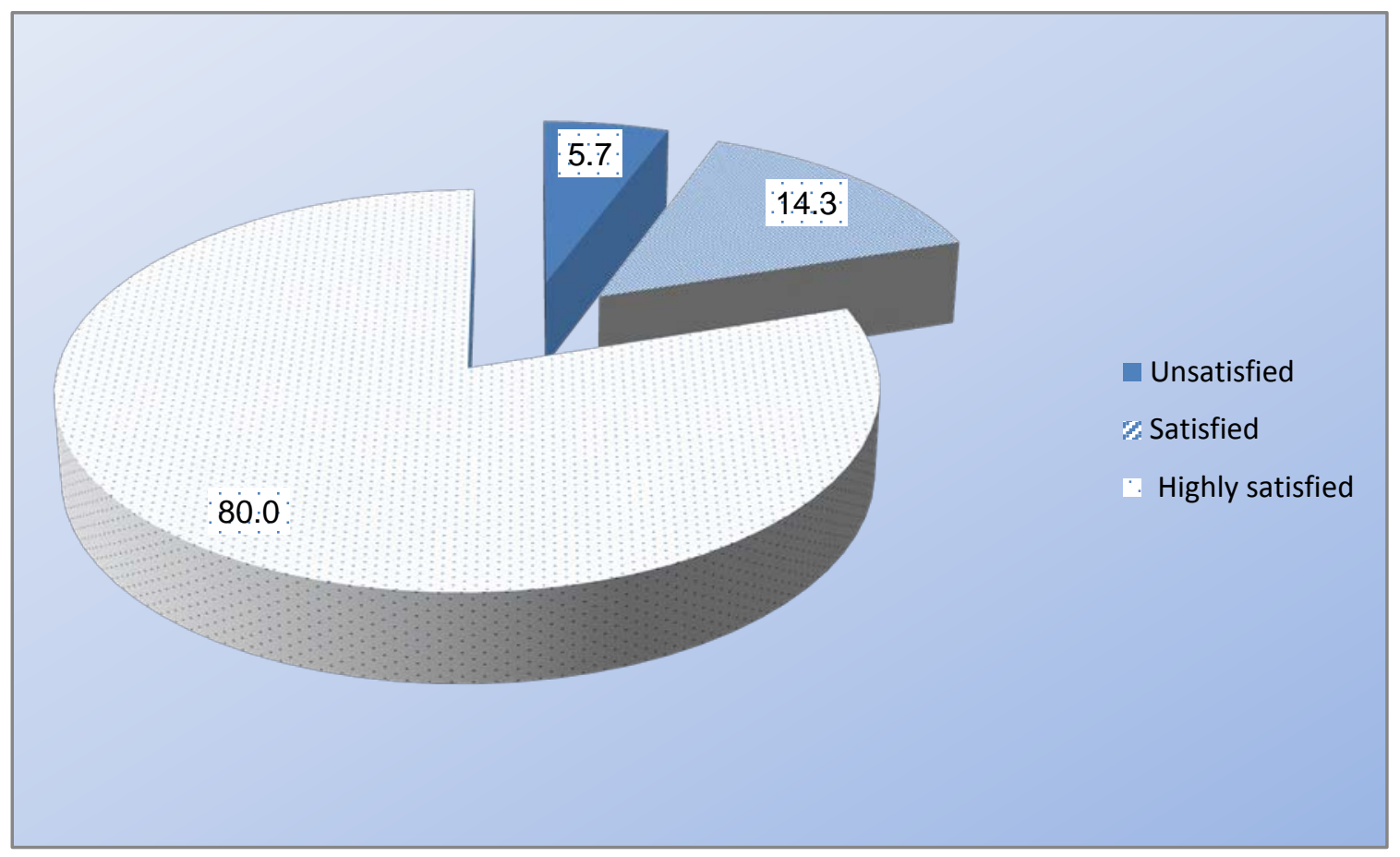

Figure 5. Frequency distribution of study group' women regarding satisfaction from antenatal instructional package (n=70)

Table 6. Correlation coefficient between total knowledge, attitude and practices scores among study and control group before instructional package implementation $(n=140)$

\begin{tabular}{|c|c|c|c|c|c|c|c|}
\hline \multirow{3}{*}{\multicolumn{2}{|c|}{$\begin{array}{c}\text { Total knowledge, attitude and } \\
\text { practices }\end{array}$}} & \multicolumn{6}{|c|}{ Before antenatal instructional package implementation } \\
\hline & & \multicolumn{3}{|c|}{$\begin{array}{l}\text { Study group } \\
\text { n-70 }\end{array}$} & \multicolumn{3}{|c|}{$\begin{array}{c}\text { Control group } \\
\mathbf{n}=70\end{array}$} \\
\hline & & $\begin{array}{c}\text { Total } \\
\text { knowledge }\end{array}$ & $\begin{array}{c}\text { Total } \\
\text { attitude }\end{array}$ & $\begin{array}{c}\text { Total } \\
\text { practices }\end{array}$ & $\begin{array}{c}\text { Total } \\
\text { knowledge }\end{array}$ & $\begin{array}{c}\text { Total } \\
\text { attitude }\end{array}$ & $\begin{array}{c}\text { Total } \\
\text { practices }\end{array}$ \\
\hline \multirow{2}{*}{ Total knowledge } & $\mathrm{r}$ & & 0.52 & 0.19 & & 0.51 & 0.61 \\
\hline & p-value & & $0.000^{* *}$ & 0.11 & & $0.000^{* *}$ & 0.17 \\
\hline \multirow[t]{2}{*}{ Total attitude } & $\mathrm{r}$ & & & 0.11 & & & 0.41 \\
\hline & $\mathrm{p}$-value & & & 0.35 & & & 0.37 \\
\hline
\end{tabular}


Table 7. Correlation coefficient between total knowledge, attitude and practices among study and control group one month after antenatal instructional package implementation $(n=140)$

\begin{tabular}{|c|c|c|c|c|c|c|c|}
\hline \multirow{3}{*}{\multicolumn{2}{|c|}{$\begin{array}{c}\text { Total knowledge, attitude and } \\
\text { practices }\end{array}$}} & \multicolumn{6}{|c|}{ One month after antenatal instructional package implementation } \\
\hline & & \multicolumn{3}{|c|}{$\begin{array}{l}\text { Study group } \\
\mathbf{n}=70\end{array}$} & \multicolumn{3}{|c|}{$\begin{array}{c}\text { Control group } \\
\mathbf{n}=70\end{array}$} \\
\hline & & \multirow{2}{*}{$\begin{array}{c}\text { Total } \\
\text { knowledge }\end{array}$} & \multirow{2}{*}{\begin{tabular}{|c|c}
$\begin{array}{c}\text { Total } \\
\text { attitude }\end{array}$ \\
0.59 \\
\end{tabular}} & \multirow{2}{*}{$\begin{array}{c}\begin{array}{c}\text { Total } \\
\text { practices }\end{array} \\
0.35\end{array}$} & \multirow{2}{*}{$\begin{array}{c}\text { Total } \\
\text { knowledge }\end{array}$} & \multirow{2}{*}{\begin{tabular}{|c|c}
$\begin{array}{c}\text { Total } \\
\text { attitude }\end{array}$ \\
0.61 \\
\end{tabular}} & \multirow{2}{*}{$\begin{array}{c}\begin{array}{c}\text { Total } \\
\text { practices }\end{array} \\
0.54\end{array}$} \\
\hline Total knowledge & $\mathrm{r}$ & & & & & & \\
\hline & p-value & & 0.06 & 0.09 & & $0.000 * *$ & 0.17 \\
\hline \multirow[t]{2}{*}{ Total attitude } & $r$ & & & 0.04 & & & 0.31 \\
\hline & p-value & & & 0.72 & & & 0.79 \\
\hline
\end{tabular}

Table 6 clarifies that, before antenatal instructional package implementation there was a highly statistical significant correlation between total knowledge and total attitude scores among both study and control group before antenatal instructional package implementation. While there was no statistically significant correlation between total knowledge and total practices score among both study and control group before antenatal instructional package implementation.Also there was no statistically significant correlation between total attitude and total practices score among both study and control group before antenatal instructional package implementation.

Table 7 displays that, there was no statistical significant correlation between total knowledge and total attitude scores among study group and highly statistical significant correlation among control group one month after antenatal instructional package implementation. While there was no statistically significant correlation between total knowledge and total practices score among both study and control group one month after antenatal instructional package implementation. Also there was no statistically significant correlation between total attitude and total practices score among both study and control group one month after antenatal instructional package implementation.

\section{Discussion}

Antenatal care provides an important opportunity for discussion between a pregnant women and a health care provider about health behavior during pregnancy and about recognizing complications that may arise during pregnancy. ANC personnel should also provide information on postpartum care, newborn care, breast feeding, signs of problems, and appropriate action to take. [13].

In this research the researchers attempted to examine the effect of antenatal instructional package on pregnant women' knowledge, attitude and practices. The findings of this research approved the research hypotheses.

Regarding socio demographic characteristics and obstetric history of the studied groups, the results revealed that there was no statistically significant difference between study and control group regarding socio demographic characteristics and obstetric history. This ensures that the two groups under the study were homogenous.

As regards the source of information about ANC, the results of current study showed that nearly one third of both study group and control groups had source of information from friends and families while minority of them had source of information from newspaper. These results agree with [17] who conducted a study to assess knowledge and attitude of antenatal women about maternal nutrition attending a tertiary care centre and found that source of information for pregnant women was mainly family members (38\%).

Knowledge is the understanding of any given topic. In the current study, it refers to a pregnant women's understanding of components of antenatal care ANC visits, smoking, alcohol intake, nutritional habits, exercises, clothes, tetanus immunization, investigations, danger signs of pregnancy, and minor disturbance with pregnancy [10].

In relation to pregnant women's knowledge during ANC, the results of the present study showed that, there was no statistical significant difference regarding all knowledge items about antenatal care between the study and the control group before instructional package implementation. However, a highly statistically significant difference ( $p$-values < 0.001) was observed regarding all knowledge items about antenatal care between the study and the control group one month after instructional package implementation. This improvement in the study group's knowledge might be due to pregnant women's active participation and good communication with the researchers who helped them to acquire knowledge. Besides, instructional package plays a very important role in helping pregnant women to acquire knowledge regarding ANC.

The result of the present study is in congruent with [22] who conduct a quasi-experimental study on effectiveness of self-instructional module on knowledge regarding antenatal care among primigravida women attending selected hospitals of Punjab and Haryana. The results showed that the intervention was useful in enhancing the knowledge about antenatal care. As well as, [23] who study the effectiveness of self-instructional module on knowledge regarding selected aspects of safe motherhood among Primigravida women in selected hospitals, reported that there was a significant improvement in knowledge of primigravida women after the administration of selfinstructional module on safe motherhood.

These results are also matched with [24], who studied the effect of health education intervention on knowledge and accessibility of pregnant women to antenatal care services in Edu, Kwara State, Nigeria and reported upward shift from pre-intervention mean of 71.08 to 93.33 postintervention mean in the experimental groups which implied the positive impact of health education intervention on the knowledge of pregnant women. Moreover, [25] who study the effect of antenatal 
educational guidelines on mother's knowledge pointed out that there was a significant improvement in antenatal knowledge level of the study group than those of the control group.

The present study revealed that there was a significant improvement of the studied women's knowledge regarding minor discomfort during pregnancy one month after instructional package implementation. This is in agreement with [26] who conduct Pre-experimental one group pretest-posttest design on sixty antenatal mothers to examine Effectiveness of IEC (Information, Education \& Communication) package on knowledge regarding minor ailments of pregnancy and its management among antenatal mothers at NMCH, Nellore, A.P. The results showed that the IEC package was effective in increasing the knowledge level of women regarding minor ailments of pregnancy. These findings are also supported by [27] who conduct a quasi-experimental study on utilization of self-care practice guideline on relieving minor discomfort (Ailments) among new pregnant woman and revealed that, there was highly statistically significant improvement knowledge mean score; knowledge mean score was increased from 11.5 to 54.59 at post intervention

As regards knowledge about danger signs during pregnancy before and one month after instructional package implementation, The present study revealed significant improvement of women's knowledge about warning signs during pregnancy in the study group one months after instructional package implementation compared to the control group. These findings are in agreement with [28] who conducted a quasi-experimental study on 120 women during their antenatal care visits at outpatient clinic in Zagazig University Hospitals to assess the effect of an educational program on pregnant women's knowledge about obstetric danger signs and results revealed that statistically significant differences were detected between total knowledge score about obstetrics danger signs at different time from health educational program implementation to the studied group.

In relation to knowledge about necessary investigations during pregnancy before and one month after instructional package implementation, The present study revealed significant improvement of women's knowledge about necessary investigations during pregnancy in the study group one months after instructional package implementation compared to the control group. This result is in agreement with [19], who conduct a cross sectional survey between February and April 2011 to assess the knowledge, attitude and practice on antenatal care among the residents in three Orang Asli settlements in Jempol District, Negeri Sembilan, namely Kampung Sungai Cherbang, Kampung Sungai Sot and Kampung Sungai Lui and results showed that the level of knowledge regarding the importance of early antenatal care and screening test were poor.

The findings of the present study illustrated that both study and control groups showed lack of knowledge regarding diet system during pregnancy before instructional package implementation. These findings are in agreement with [17], who conduct a cross sectional study on 850 antenatal women from August 2015 to February 2016 to assess knowledge and attitude of antenatal women about maternal nutrition attending a tertiary care centre. The results reported that only $22 \%$ of the women had good knowledge about nutrition during pregnancy and $82 \%$ showed willingness to acquire more information about maternal nutrition. On the other hand, the present study revealed that there was a significant improvement of the studied women's knowledge regarding diet system during pregnancy one month after instructional package implementation

Attitudes are emotional, motivational, perceptive and cognitive beliefs that positively or negatively influence the behavior or practice of an individual [29].

Concerning pregnant women's attitude during ANC, the results of current study showed that, there was no statistical significant difference regarding all attitude items about antenatal care between the study and the control group before instructional package implementation. However, a highly statistically significant difference (p-values < 0.001) was observed regarding all attitude items about antenatal care between the study and the control group one month after instructional package implementation. The difference in health attitude score of pregnant women as reported can be linked to antenatal instructional package which is a more inclusive teaching as compared to usual health education given at antenatal clinic.

These findings are in agreement with [1] who examines the effect of maternal health education on the health attitude of pregnant mothers attending ante-natal clinics in Anambra State, Nigeria. The result of the study showed effect of maternal health education on health attitude of mothers. The experimental group recorded a higher mean difference health attitude score $(X=11.93)$ than the control group $(\mathrm{X}=1.23)$. Hence, maternal health education enhanced pregnant mothers' health attitude positively.

As well as, [30], who evaluate the effectiveness of selfinstructional module on knowledge and attitude regarding antenatal care among primigravida mothers in selected government hospital, Dehradun, Uttarakhand reported that the mean attitude score of pregnant women before intervention was 20.50 and the attitude level after intervention was 23.84 with statistical significant difference.The result findings disclose that there was an improvement in the attitude level among pregnant women regarding antenatal care.

Practices are defined as the observable actions of a pregnant woman that could affect her to go to the hospital for antenatal check-up [31]. Regarding pregnant women's practices during ANC, the results of the present study mentioned that, there was no statistical significant difference regarding all practice items about antenatal care between the study and the control group before instructional package implementation. However, a highly statistically significant difference ( $p$-values $<0.001$ ) was observed regarding all practice items about antenatal care between the study and the control group one month after instructional package implementation. This improvement in study group's practices might be due to the fact that the pregnant women recognized the importance and availability of carrying out all the recommended instructions in the instructional package.

These results are in accordance with [32] who examine effect of counseling intervention on women's knowledge, practices and lifestyle of fetal well-being among primigravida. The results showed that the majority of the 
women (96.6\%) follow healthy life style practices after application of the intervention. In addition, [33] who investigate the effect of an educational intervention on pregnancy: a cluster-randomized clinical trial, found that there was an increase in the prevalence of attitudes, and practices regarding pregnancy in the intervention group.

Satisfaction of pregnant women seeking help, is one of the most important qualitative indexes of health care provision, and has a very special importance in antenatal care. Satisfying pregnant women is achieved through satisfying their needs and expectations [12].

Regarding satisfaction from ANC instructional package, the results of the current study revealed that more than two thirds of study group' women had highly satisfaction from the antenatal instructional package. This result agrees with [24], who reported that pregnant women's satisfaction over the health education package delivered during their ANC visits.

Concerning correlation coefficient between total knowledge, total attitude and total practices scores, the results showed that before antenatal instructional package implementation there was a highly statistical significant correlation between total knowledge and total attitude scores among both study and control group. While there was no statistically significant correlation between total knowledge and total practices score among both study and control group before antenatal instructional package implementation. Also there was no statistically significant correlation between total attitude and total practices score among both study and control group before antenatal instructional package implementation.

On the other hand, the results showed that there was no statistical significant correlation between total knowledge and total attitude scores among study group and highly statistical significant correlation among control group one month after antenatal instructional package implementation. In the researchers point of view this ensure that pregnant women in the study group acquired correct knowledge which in turn affect their attitude positively. While there was no statistically significant correlation between total knowledge and total practices score among both study and control group one month after antenatal instructional package implementation. Also there was no statistically significant correlation between total attitude and total practices score among both study and control group one month after antenatal instructional package implementation

These findings are supported by [34] who study Knowledge, attitude, and practices of pregnant women towards antenatal care in primary healthcare centers in Benghazi, Libya and mentioned that the level of overall knowledge had a significant direct correlation with the practices towards antenatal care $(\mathrm{r}=0.228, \quad \mathrm{P} \leq 0.001)$, whereas it had an insignificant correlation with the attitude $(r=0.029, \mathrm{P}=0.619)$.

\section{Conclusion}

Based on the findings of the current study; the study concluded that research hypotheses are supported and the antenatal instructional package had appositive effect on improvement of pregnant women' knowledge and in turn attitude and practices were also improved in study group compared to control group and this support the first hypothesis. Also the majority of pregnant women in the study group were satisfied with the antenatal instructional package implementation and this support the second hypothesis.

\section{Recommendations}

Based on the findings of the current research, the following recommendations are suggested:

- From time to time, the antenatal instructional package should be restructured and reviewed to meet up with the health needs of the pregnant women.

- From the finding that antenatal instructional package is significant in influencing maternal knowledge, health attitude and health practices; the researchers recommended that antenatal instructional package must be seriously upheld in every antenatal clinic.

- Pregnant women satisfaction surveys should be carried out routinely to improve the quality of antenatal care services.

Further research:

- Replication of the study on large representative probability sample is highly recommended in different maternity hospitals to achieve more generalization of the results.

- Further studies should be conducted to incorporate the views and responses of health care providers, as the study only depended on the responses of pregnant women.

\section{References}

[1] Nwankwo, C.U., Okafor, J.O., Makachi, M.C., Anieche, J.E., Chiejina, E.N., et al., (2016). Effect of maternal health education on the health attitude of pregnant mothers attending ante-natal clinics in Anambra State, Nigeria. IOSR Journal of Nursing and Health Science. 5(1): 56-63.

[2] Shafqat, T., Fayaz, S., Rahim, R., and Saima, S., (2015). Knowledge and awareness regarding antenatal care and delivery among pregnant women. J Med Sci 23(2): 88-91.

[3] Pierre-Marie, T., Gregory, H., Maxwell, D.I., Robinson, E.M., Yvette, M., et al., (2015). Maternal mortality in Cameroon: A university teaching hospital report. Pan African Medical Journal. 2015; 21:16. Available at: http://www.ncbi.nlm.nih.gov/pmc/ articles/PMC4561158/. Accessed September, 23 2018, 10Am.

[4] Bande, M. I., Chutiyami, M., Shehu, U., and Garba, M. S., (2018). Effects of Socio Demographic and Institutional Factors on Utilization of Antenatal Care Services among Pregnant Women in Damaturu, Yobe State, Nigeria. International Journal of tropical disease \& Health. 29(2): 1-9.

[5] Muyunda, B., Makasa, M., Jacobs, C., Musonda, P., and Michelo, C., (2016). Higher Educational Attainment Associated with Optimal Antenatal Care Visits among Childbearing Women in Zambia. Front. Public Health 4:127.

[6] World Health Organization. (2017). World Health Statistics. (2017). Available at: www.who.int/gho/publications/world_health_statistics/2014/en/. Accessed August, 20 2018, 11Am.

[7] Wolderufael, T.S., (2018). Factors Influencing Antenatal Care Service Utilization Among Pregnant Women in Pastoralist Community in Menit-Shasha District, Ethiopia.Int J Med Res Health Sci 2018, 7(5): 143-156.

[8] Bank, T.W., (2013). Trends in Maternal Mortality: 1990 to 2013. 
[9] Al hazmi, J.M., Habib, H.M., Sebeih, S.H., Khan, M.I., Elmaghrabi, S.A., et al., (2017). Awareness of Antenatal Care Importance among Saudi Pregnant. Journal of Gynecology and Women's Health. 4(4): 1-15.

[10] Patel, B.B, Gurmeet, P., Sinalkar, D.R., Pandya, K.H., Mahen, A., et al., (2016). A study on knowledge and practices of antenatal care among pregnant women attending antenatal clinic at a Tertiary Care Hospital of Pune, Maharashtra. Medical Journal. 9(3): 354-362.

[11] Lilungulu, A.G., Matovelo, D., and Gesase, A., (2016). Reported knowledge, attitude and practice of antenatal care services among women in dodoma municipal, tanzania. Journal of Pediatrics and Neonatal Care.2016, 4(1): 00125

[12] Al-Ateeq, M., and Al-Rusaiess, A., (2015). Health education during antenatal care. International Journal of Women's Health. 2015:7 239-242.

[13] Almalik, M.M.A., and Mosleh, S.M., (2017). Pregnant women: What do they need to know during pregnancy? A descriptive study. Women and birth: journal of the Australian College of Midwives, 30(2): 100-106.

[14] Alshabanah, R.F., Almohayya, T.S., and Alahmari, E.M., (2018):Assessment of Knowledge, Attitudes and Practices of Expectant Mothers in Relation to Antenatal Care in Abha. The Egyptian Journal of Hospital Medicine. 70 (6): 939-942.

[15] World Health Organization, (2014). Maternal mortality fact sheet. Available at: http://apps.who.int/iris/bitstream/handle/10665/112318/WHO_RH R_1. Accessed July, 15 2018, 11pm.

[16] Nwaneri A.C., Ndubuisi, I., Okoronkwo, I.L., Ezike, O., and Nkiruka, U., (2018). Determinants of late booking for antenatal care among pregnant women in selected hospitals in South East Nigeria. International Journal of Nursing and Midwifery. 10(7): 74-80.

[17] Gupta, R., Agarwal, S., Singh, N., Jain, R., Katiyar, A., et al., (2016). A study to assess knowledge and attitude of antenatal women about maternal nutrition attending a tertiary care centre. International Journal of Reproduction, Contraception, Obstetrics and Gynecology, 5(5):1549-1552.

[18] Divya, M., Pushpa, N., Lata, R., Iqbal, R., and Nidhi, S., (2017). Knowledge, attitude and practices of antenatal care among pregnant women attending antenatal clinic at SMS Medical College Jaipur. International Journal of Science and Applied Research, 4(7): 08-12.

[19] Rosliza, A.M., and Muhamad, H.J., (2011). Knowledge, attitude and practice on antenatal care among Orang Asli women in Jempol, Negeri Sembilan. Malaysian. Journal of Public Health Medicine, 11(2): 13-21.

[20] Nun, D.R., Adesuyi, E.O., and Olawoore, S.A., (2018) Knowledge attitude and practices of pregnant women attending comprehensive Health Centre, Isolo, Ondo State towards Hygienic Practice. International Journal of TROPICAL DISEASE \& Health. 30(2): 1-10.

[21] Singer, A.J., and Thode, J.R., (1998). Determination of the minimal clinically significant difference on a patient visual analog satisfaction scale. Acad Emerg Med. 5: 1007-11.

[22] Kaur, H., Ramanadin, P., Vadivukkarasi, and Bala, K., (2015). A quasi experimental study on effectiveness of self-instructional module on knowledge regarding antenatal care among primigravida women attending selected hospitals of Punjab and Haryana. Int J Psychiatric Nurs. 1: 188-94.

[23] Indra, V., (2016). Effectiveness of Self Instructional Module (SIM) on Knowledge Regarding Selected Aspects of Safe Motherhood among Primigravida Women in Selected Hospitals, Puducherry. International Journal of Nursing Education and Research. 4(3): 2454-2660.

[24] Jibril, U.N., Saleh, G.N., Badaki, O., Anyebe, E.E., Umar, A., et al., (2018). Health education intervention on knowledge and accessibility of pregnant women to antenatal care services in Edu, Kwara State, Nigeria. International Journal of Women's Health and Reproduction Science. 6(2):154-160.

[25] Abdel Hafez, A.A., Makhlouf, E.M., and Mohammed, O., (2018). Effect of antenatal educational guidelines on mother's knowledge. IOSR Journal of Nursing and Health Science. 7(2):21-26.

[26] Latha, P., and Indira, S., (2016). Effectiveness of IEC (Information, Education \& Communication) package on knowledge regarding minor ailments of pregnancy and its management among antenatal mothers at NMCH, Nellore, A.P.International Journal of Applied Research. 2(10):593-596.

[27] Abd Elhaliem, S., AbdElhady, R., and Mohamed, A., (2018):Utilization of Self-Care Practice Guideline on Relieving Mino Discomfort (Ailments) Among New Pregnant Woman. IOSR Journal of Nursing and Health Science. 7(1): 07-15.

[28] Mohamed, N.S., (2016). The effect of an educational program on pregnant women's knowledge about obstetric danger signs. IOSR Journal of Nursing and Health Science, 8(1): 08-16.

[29] Vainikka, P., (2015). Psychological factors influencing consumer behaviour. Bachelor's Thesis. Centria University of Applied Sciences. Pp27:28.

[30] Nidhi, J., (2018). Effectiveness of self-instructional module on knowledge and attitude regarding antenatal care among primigravida mothers in selected government hospital, Dehradun, Uttarakhand. International Journal of Medical Science and Public Health. 7(8):651-654.

[31] Bej, P., (2018). Knowledge, attitude and practices among pregnant women about antenatal care, danger sign during pregnancy and adopting family planning method.4 (1):1-10.

[32] Oliveira, S.C., Fernandes, A.F., Vasconcelos, E.M., Ximenes, L.B., Leal, L.B., et al., (2018). Effect of an educational intervention on pregnancy: a cluster-randomized clinical trial. Acta Paulista de Enfermagem. 31(3):8-291.

[33] Said, A.R., (2016). Effect of counseling intervention on women's knowledge, practices and lifestyle of fetal well-being among Primigravida. International Journal of Nursing Science. 6(4): 87-93.

[34] Ibrahim, H.K., El Borgy, M.D., and Mohammed, H.O., (2014) Knowledge, attitude, and practices of pregnant women towards antenatal care in primary healthcare centers in Benghazi, Libya.J Egypt Public Health Assoc. 89(3): 26-119. 